\title{
Near-infrared spectroscopy for the prediction of auxiliary quantities to characterize the product quality of a novel cementitious material
}

\author{
C. Lutz ${ }^{1}$, J. Matthes ${ }^{1}$, P. Waibel ${ }^{1}$, U. Precht ${ }^{2}$, U. Schweike ${ }^{2}$, \\ G. Beuchle ${ }^{2}$, K. Garbev ${ }^{2}$, P. Stemmermann ${ }^{2} \&$ H. B. Keller ${ }^{1}$ \\ ${ }^{1}$ Institute for Applied Computer Science, \\ Karlsruhe Institute of Technology (KIT), Germany \\ ${ }^{2}$ Institute for Technical Chemistry, \\ Karlsruhe Institute of Technology (KIT), Germany
}

\begin{abstract}
The production of cement is one of the most energy and $\mathrm{CO}_{2}$ intensive industries. At the Karlsruhe Institute of Technology a new "green" process for the production of cementitious materials was developed with a high potential for saving energy and $\mathrm{CO}_{2}$-emissions. In a two-stage process a new hydraulic binder (Celitement) comparable to Portland cement is produced from limestone and sand. Until now, the product was only analysed with complex analytical methods. In the future it is necessary to have a robust measurement system, which is able to determine the product quality online during its manufacture. In this paper, the development of a method for the prediction of auxiliary quantities for characterization of the product quality based on an online near-infraredspectroscopy (NIR) is presented. Initial methods with data pre-processing and partial-least-squares-regression for systematic development of near-infrared calibration models and their application to new data sets are presented. In addition, a new method for data pre-processing by adapting the target vector to the working point in comparison to the calculation of calibration models with absolute values is shown.

Keywords: Celitement, cementitious material, NIR-spectroscopy, calibration model, partial-least-squares-regression.
\end{abstract}




\section{Introduction}

With a share of $5-8 \%$ to the global $\mathrm{CO}_{2}$ emissions the production of cement is one of the industries with the highest contribution to anthropogenic greenhouse gases [1]. Additionally, there is a significant increase in cement production in the recent decade, especially in Asia. At the Karlsruhe Institute of Technology a new process for manufacture of the novel hydraulic binder named Celitement was developed. The production of Celitement allows for reduction of $\mathrm{CO}_{2}$-emissions by $50 \%$ compared to that of the ordinary Portland cement (OPC). The novel process consists of two steps: a hydrothermal process with subsequent drying and an activation treatment. A pilot plant with a capacity of $100 \mathrm{~kg}$ Celitement per day was commissioned and built in 2011.

However, so far it is difficult to determine the product quality during the process based on analytical techniques such as $\mathrm{X}$-ray diffraction (XRD) and $\mathrm{X}$-ray fluorescence (XRF), which are otherwise well established for production control of OPC. This is mainly due to the amorphous nature of both precursor and final product. Minor crystalline constituents can still be quantified by XRD analysis with the Rietveld method. The major amorphous components are accounted for via a combination of thermogravimetric analysis and spectroscopic methods. At the moment, it is difficult to apply such analytical techniques during the process (online). In order to reduce the effort and to allow online measurements during system operation, various auxiliary quantities for characterization of the product quality have to be defined. As an analytical method of choice, measurement of the auxiliary quantities with NIR-spectroscopy was foreseen because of its simplicity in terms of performance, robustness and easiness of quantification. This work gives an overview of the Celitement production process and the issues related to the fast assessment of the product quality. Subsequently, different auxiliary quantities for determination of the product quality and methods for a systematic development of calibration models based on NIR-spectra and their application to new data sets are derived. Additionally, a new method for data pre-processing by adapting the target vector to the working point is compared with the calculation of the calibration model from absolute values. Finally, an example of two Celitement data sets will illustrate the ideas and methods which build the basis of this study.

\subsection{Celitement}

The adhesive components in the hardened cement paste consist mostly of calcium silicate hydrates (C-S-H phases), which are responsible for the mechanical strength and chemical resistance of concrete and mortar. Upon hydration of OPC only about $50 \%$ of all reaction products are the desired C-S-H phases. This is mainly due to the very high $\mathrm{CaO} / \mathrm{SiO}_{2}$ ratio of OPC $(\sim 3.5)$. The remaining $50 \%$ consist of calcium hydroxide and sulphate and/or carbonate containing calcium aluminate hydrates known generally as AFt and AFm phases. These by-products can negatively influence the mechanical properties and resistance of the concrete against different kinds of chemical attack (carbonation, 
delayed ettringite formation etc.). In order to obtain a higher yield of C-S-H phases upon hydration of cement, it is necessary to decrease the calcium content, which is not possible because the existing clinker technology is based on production of minerals rich in $\mathrm{Ca}$ (mainly $\mathrm{Ca}_{3} \mathrm{SiO}_{5}$ and $\mathrm{Ca}_{2} \mathrm{SiO}_{4}$ ). Nowadays the cement industry tries to reduce the $\mathrm{Ca}$ content by mixing conventional clinker with latent hydraulic materials like blast furnace slag, fly ash, microsilica, etc.

Preferably an ideal hydraulic binding material should be able to be transformed completely into C-S-H phases upon hydration. In the early stages of hydration of Portland cement a partially hydroxylated intermediate product with low calcium content was discovered. The Celitement process includes the formation of a similar hydroxylated precursor with significantly lower calcium content in comparison to the Portland cement production. (See $[2,3]$.

Figure 1 shows the concept of the Celitement production, which is based on similar raw materials as OPC. The starting materials calcium oxide from calcinated calcium carbonate and silicon oxide are mixed in $\mathrm{CaO} / \mathrm{SiO}_{2}$ ratio $<2$ and treated hydrothermally at temperatures between $140^{\circ} \mathrm{C}$ and $300^{\circ} \mathrm{C}$.

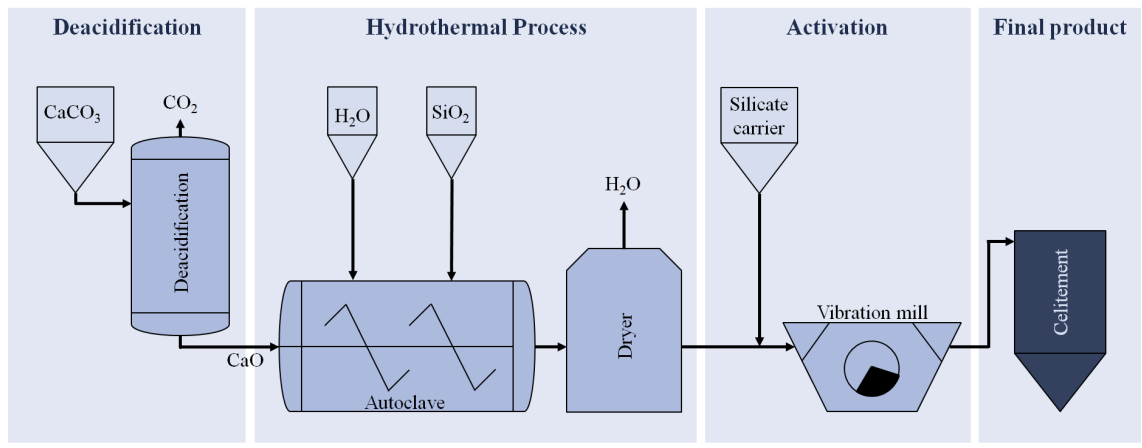

Figure 1: Procedure of the Celitement process.

In the hydrothermal process C-S-H phases are formed, which are stabilized by hydrogen bonds with silanol groups and are consequently not hydraulically active. In the subsequent drying stage excess water is removed. For the hydraulic activation of the C-S-H precursor the existing hydrogen bond system needs to be modified. For this purpose, the hydrothermally produced precursor is mixed and ground with a silicon oxide carrier. As a result a structural reorganization including amorphization and partial breakage of the hydrogen bond system takes place. The new formed fragments consisting of calcium hydrosilicates are accumulated on the silicate surfaces and form a reactive shell over the inert core. In contrast to the conventional cement production, Celitement contains up to $8 \%$ $\mathrm{H}_{2} \mathrm{O}$ which plays an important role in the bonding scheme of the resulting $\mathrm{C}-\mathrm{S}-\mathrm{H}$ phases. Overall, only about a third of calcium oxide is needed for the reaction, which corresponds to a reduction of $\mathrm{CO}_{2}$ emissions with up to $2 / 3$ in comparison with OPC. (See [2-4].) 


\subsection{NIR-spectroscopy}

NIR-spectroscopy is a fast and non-destructive technique which is increasingly used in process probes in food and agricultural as well as chemical, pharmaceutical and petrochemical industries. The most important advantages of NIR-spectroscopy are the simple sample preparation, the possibility for rapid on-line measurements with fiber optics and simultaneous multi-component analyses using chemometric methods for determination of desired parameters. A broad range of both physical and chemical information can be extracted from NIR-spectra. NIR-spectroscopy is based on the effect of the interaction between matter and electromagnetic radiation (mostly generated by a tungsten lamp) which stimulates the polyatomic molecules to oscillation and rotation. The near-infrared region of the electromagnetic spectrum spans the range $12.500-4.000 \mathrm{~cm}^{-1}(800-2500 \mathrm{~nm})$ and is dominated by overtones and combinations of $\mathrm{O}-\mathrm{H}(\mathrm{N}-\mathrm{H}, \mathrm{C}-\mathrm{H}$ etc.) rather than fundamental vibrations which are seen mostly in the far- and mid-infrared regions.

The radiation transmitted through the sample, respectively the reflected radiation in relation to the initial radiant power is measured as a function of energy. There are different methods for NIR measurements including common transmission or reflection techniques. Due to the weakness of the combination and overtone bands, which stay in relation 1/10 to $1 / 100$ to the fundamentals, the NIR method can only be applied to solids and liquids. On the other hand the samples do not need to be diluted which additionally simplifies the sample preparation.

Although NIR offers the greatest diversity of instrumentation principles among all optical spectroscopic methods, the use of Fourier-transform-infraredspectrometer (FTIR-spectrometer) proved to be the best choice regarding the quality of the spectra obtained. It is due to the inherit properties of the FTIR-spectrometry which is distinguished by its outstanding efficiency (no need of monochromator slit, Jaquinot advantage), accuracy (in terms of spectral frequency guaranteed by precise interferogram triggered by He-Ne Laser) and high scan speed (0.01-1s) which allows a higher signal-to-noise ratio and shorter measurement periods. (See [5-7].)

\section{Experimental setup}

Currently, the product quality of Celitement is controlled either by the determination of the mechanical strength on standard prisms of mortars after EN 196 or by combined analytical methods, comprising XRD, thermogravimetric analysis and vibrational spectroscopy. In future, it should be possible to approximate the product quality on auxiliary quantities already during the manufacturing process. In the first instance, reliable auxiliary quantities must be defined and determined. Subsequently, a data set of samples for different process states has to be analysed in relation to the auxiliary quantities and the NIRspectra. 


\subsection{NIR-spectra}

The characterization of Celitement samples was performed with an FTNIR-spectrometer MATRIX-F (Bruker Optics, Ettlingen, Germany). It is particularly suitable for dusty environments and temperature fluctuations that may occur during the Celitement production. In addition, the device is real-time capable and it is possible to transfer methods developed in the laboratory directly to the process application. The NIR-spectra in diffuse reflectance mode were recorded in the range $4000-12000 \mathrm{~cm}^{-1}$ with a resolution of $8 \mathrm{~cm}^{-1}$ and the average of 32 scans with a handheld reflection probe IN261 (Bruker Optics, Ettlingen, Germany). (See [8].)

Before the acquisition of the spectrum, the material was mixed in the sample container and compacted. Then, the reflection probe was immersed into the sample and the scan was started while a small amount of pressure was exercised on the sample. For each sample three spectra were taken by repeatedly mixing, compacting and immersing.

To calculate the calibration model for defined auxiliary quantities, different NIR-spectra were recorded on samples produced under different process conditions. In addition, different batches with theoretically same initial composition should be considered in order to obtain data sets with a wide variation of the auxiliary quantities. The compositions of different batches may vary due to fluctuations in raw material composition and different process settings. Therefore, it is necessary to supplement the calibration model continuously with new data sets in order to achieve a complete and robust model considering different situations and compositions.

\subsection{Auxiliary quantities for the characterisation of the product quality}

Besides the strength analysis, the previous analyses and inspections of the product quality were mainly based on the XRD with the Rietveld method. With the XRD it is possible to identify and quantify the crystalline components contained in the material as well as the crystallite sizes and the fraction of $\mathrm{X}$-ray-amorphous constituents. Initially, three different measurements determined by XRD are selected for the auxiliary quantities calibration:

- Alpha: The fraction of $\alpha-\mathrm{Ca}_{2}\left[\mathrm{HSiO}_{4}\right] \mathrm{OH}\left(\alpha-\mathrm{C}_{2} \mathrm{SH}\right)$ in the sample, which is a product in the autoclave process.

- SizeQuartz: Refers to the average crystallite size of the quartz crystals contained.

- Amorphous: The fraction of $\mathrm{X}^{-}$-ray-amorphous material in the sample.

Alpha and SizeQuartz can give an indication of the progress of the grinding process. Presence of Alpha gives rise to sharp NIR bands at $4200-4300 \mathrm{~cm}^{-1}$ while sizeQuartz has only indirect effects on the NIR spectra. The hydraulically active components of Celitement are amorphous and thus included in the overall $\mathrm{X}$-ray-amorphous fraction. Therefore, it is particularly interesting to involve this indicator for the estimation of the product quality. Amorphous C-S-H is represented in the NIR spectra by broad bands between $4750-5450 \mathrm{~cm}^{-1}$ as well as $5450-6000 \mathrm{~cm}^{-1}$. In the final stages the fraction of the amorphous components 
should be preferably high and the portion of Alpha should be as low as possible, as Alpha doesn't contribute to the strength of the concrete.

\section{Methods for development of calibration model}

For further treatment the spectra were exported to MATLAB R2013b (MathWorks Inc, USA). Subsequently, the processing of the data and the calculation of the calibration model could be executed. For this purpose, an algorithm was developed to calculate the calibration models for different combinations of data pre-processing, different ranges in the spectra and several model orders $a$. The calculation and evaluation of the calibration model was carried out with partialleast-squares-regression (PLSR) and different quality criteria. The quality criteria specify the selection of the most appropriate data pre-processing, the best combination of the wavenumber ranges and the optimal model order. The training data set is divided into a matrix with raw data of the NIR-spectra $\boldsymbol{X}_{\text {cal }} \in$ $\mathbb{R}^{M x N}$, a column vector $\boldsymbol{y}_{c a l} \in \mathbb{R}^{N}$ containing the selected auxiliary quantity and a wavenumber vector $\boldsymbol{\omega}$, where $M$ is the number of features (wavenumbers) and $N$ the number of samples respectively the number of recorded NIR-spectra.

\subsection{Data pre-processing}

A pre-treatment of the NIR-spectra was applied in order to reduce disturbing effects such as noise and to distinguish the desired characteristics. The calibration model was calculated for all combinations of data pre-processing steps. Using the quality criteria the best combination was selected. It must be distinguished between mandatory and optional data pre-processing. For the mandatory data pre-treatment, which is required for the implementation of PLSR, two possibilities were used:

- Column centring, in which the spectra are mean-adjusted for each wavenumber and

- Multiplicative scatter correction (MSC), where the average spectrum of the data set is determined and each spectrum is adjusted to this average spectrum by linear stretching and shifting with the method of least squares [5].

For the optional data pre-treatment the following optional data pre-processing methods can be combined in different variations.

- The moving average filter can reduce fluctuations that superimpose the trend.

- The first derivative spectrum can help to remove baseline effects and to increase the spectral resolution.

- In most cases it is also reasonable to limit the data to a certain wavenumber range. For instance, in the regions of very large and very small wavenumbers the spectra often contain a very high amount of noise, which yield virtually no spectral information.

- The standard normal variate (SNV) transformation can be applied for additional correction of scattering effects caused by variation of particle size. 
To calculate the calibration model with PLSR, it is also necessary to mean-adjust the target vector with the auxiliary quantities analogous to the mandatory data pre-processing of the spectra. (See $[5,9,10]$.)

\subsection{Subdivision of the spectrum into wavenumber ranges}

For the estimation of the respective auxiliary quantity, it is not necessary to use the whole spectrum in the calibration. It could be subdivided into several ranges. The number and size of these ranges can be selected arbitrarily and can then be included or omitted in the calculation of the calibration models. On the one hand the selection of the ranges can be chemically motivated by the presence of characteristic absorption bands in the spectrum. On the other hand, ranges could be selected in order to avoid regions of noisy data. In order to get the best combination of spectral ranges, the calibration models were calculated for all permutations of the subdivided ranges. Subsequently, each model could be evaluated by application of the quality criteria.

\subsection{Adaptation of the working point for the target vector}

In addition to the described data pre-processing, the target variable can also be normalized for the calculation of the calibration model. The absolute values $y_{i}$ can be transferred to the relative change $y_{i, \%}$ with respect to the initial value $y_{0}$ as the working point and the time of the processing step. This approach is only possible for series of measurements with known initial values of the target variables (auxiliary quantities). In case of Celitement this specification is given by the inherent batch process operation and the analysis of the intermediates. The auxiliary quantities were converted to a percentage of deviation with

$$
y_{i, \%}=\frac{y_{i}-y_{0}}{y_{0}}
$$

from the initial value (working point) and the absolute value $y_{i}$. For the prediction with the application of the calibration model to a new data set this percentage of relative change can then be recalculated with the known initial value to an absolute value. The advantage of this new data pre-processing method is the resulting adaptation of the target vector to the working point. Thus, the change related to the initial composition is used for calibration. Subsequent application of this calibration to new data sets allows prediction of a given auxiliary quantity even for compositions outside the calibration region, provided that the relation of the relative values fits.

\subsection{Partial-least-squares-regression}

Within the mathematical evaluation of the NIR-data for the Celitement process the focus was on the standard procedure of iterative PLSR. For this purpose, a linear model approach

$$
\boldsymbol{y}_{\text {cal }}=\boldsymbol{X}_{\text {cal }} \boldsymbol{b}_{1}+\mathbf{1}_{N} b_{0}+\boldsymbol{e}
$$

was selected, where $\boldsymbol{b}_{1}$ and $b_{0}$ represent the calibration model and $\boldsymbol{e}$ is a zeromean Gaussian noise vector representing the deviations from the model and 
random errors (noise). With least-squares minimization it is possible to transfer this problem into

$$
\|\boldsymbol{X} \boldsymbol{b}-\boldsymbol{y}\|_{2}^{2} \stackrel{!}{=} \min _{\boldsymbol{b}}
$$

with $\boldsymbol{X}$ as column-centred calibration matrix and $\boldsymbol{y}$ as column-centred target vector. According to the least-squares method, equation (3) is resolved for vector $\boldsymbol{b}$ by

$$
\boldsymbol{b}_{L S}=\left(\boldsymbol{X}^{T} \boldsymbol{X}\right)^{-1} \boldsymbol{X}^{T} \boldsymbol{y}
$$

in order to obtain the minimal sum of squared errors. However, this equation is only solvable, if $\boldsymbol{X}$ has full column rank. The present calibration data sets have significantly more features $N$ (to the wavenumbers corresponding absorption measurements) than measurements of different samples $M$ can be performed. One opportunity to solve the problem is the reduction of dimension from $\boldsymbol{X}$ to a model order $a \leq N$ using a suitable weighting matrix $\boldsymbol{W} \in \mathbb{R}^{M x a}$ referred to the scores matrix $\boldsymbol{T}=\boldsymbol{X} \boldsymbol{W}$. The overall problem for the calibration model can then be solved by iterative PLSR and the equation

$$
\boldsymbol{b}=\boldsymbol{W}\left(\boldsymbol{W}^{T} \boldsymbol{X}^{T} \boldsymbol{X} \boldsymbol{W}\right)^{-1} \boldsymbol{W}^{T} \boldsymbol{X}^{T} \boldsymbol{y} .
$$

(See $[11,12]$.

\subsection{Validation and selection of the calibration model}

The spectra are subdivided into four equal parts of wavenumber ranges and all variations (permutations) of involving and omitting the wavenumber ranges were tested. Additionally, the calibration models were calculated for all combinations of the pre-processing steps for every model order $a$ up to the selected maximal order (the maximal possible model order is the number of samples integrated in the calculation). Eventually, the best model should be selected from all calculated models. Figure 2 gives an overview of the whole procedure.
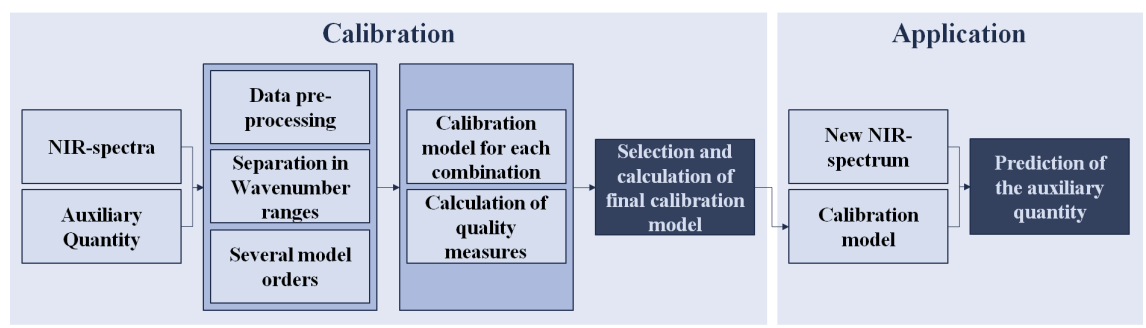

Figure 2: Procedure for calculation and selection of the calibration model.

The cross-validation was applied as an internal validation process in which a certain number of samples were not used for calibration but only for validation. After each sample was left out once for the calculation of the calibration model, the different quality criteria:

- coefficient of determination $\left(\mathrm{R}^{2}\right)$,

- root mean square error of cross validation (RMSECV) and

- residual prediction deviation (RPD). 
are calculated for the evaluation of the calibration model solely based on validation data. (See [9, 12].)

For the selection of the data pre-processing steps and the selection of the spectral ranges the combination with the highest level of $\mathrm{R}^{2}$ is selected. With respect to the prediction error and the robustness of the model, also the selection of the model order plays a decisive role. The target variable should be described as accurately as possible while keeping the amount of redundant information and the complexity of the model as low as possible.

\section{Results}

Two sets (A and B) of calibration samples were created by grinding two similar hydrothermal precursors as a function of time in order to simulate the process duration. The samples were analysed by both recording the NIR-spectra and determining the corresponding auxiliary quantities by XRD analysis as outlined above. Calibration models were calculated for all auxiliary quantities for both sets independently. In a further step the calibration models obtained were applied on the complementary sets, i.e. the calibration of set A was tested on the set B and vice versa. The results for the auxiliary quantity Alpha will be presented in the following. One important issue is also the comparison between the calibration models calculated with absolute values and relative values with respect to the working point.

Figure 3(a) shows the progress of Alpha with increasing process duration and Figure 3(b) shows the corresponding raw data of the recorded NIR-spectra for measurement series A. It can be seen that the ratio of Alpha decreases with increasing process duration for both measurement series in spite of the different initial value.

Figure 4(a) presents the results of the cross-validation for the selected calibration model with the best pre-processing methods, the best combination of spectral ranges and the associated best model order for measurement series A. The best pre-processing for this measurement series and auxiliary quantity Alpha is the first derivative of the moving average filtered spectra with standard normal variate transformation for the optional pre-processing and column centring as mandatory data pre-processing. The best result was achieved with the selection of the spectral range from $8.015 \mathrm{~cm}^{-1}$ to $6.113 \mathrm{~cm}^{-1}$ and a model order of two. With these combinations for the calculation of the calibration model by PLSR and cross-validation a $\mathrm{R}^{2}$ of about $98.8 \%$ and a relative error in average of $3.09 \%$ was achieved.

If the calibration model is now applied to measurement series B (Figure 4(b)), noticeable differences between the measured and calculated values with a relative error of $134.45 \%$ in average occur. This illustrates that the utilization of the absolute values for the calculation of the calibration model and subsequent application to new spectra for prediction of Alpha does not deliver meaningful results. Due to the different initial composition of the sample series, the values of the measured variable Alpha lie in different regions for the two 


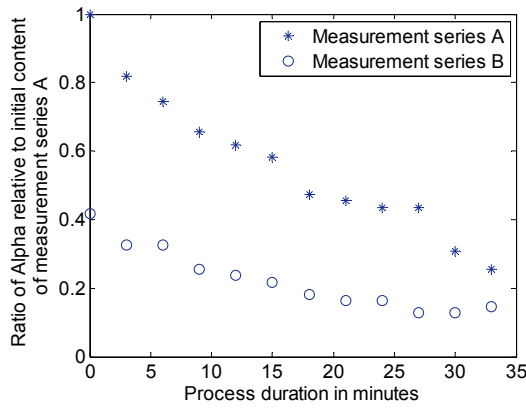

(a)

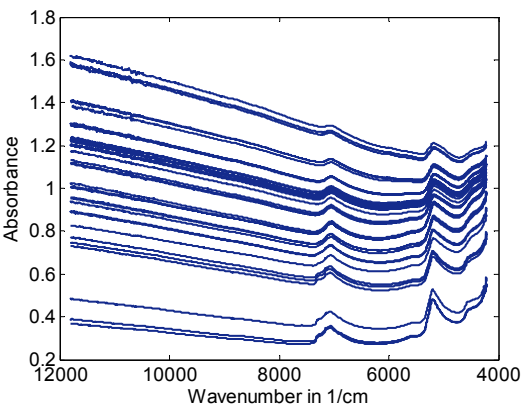

(b)

Figure 3: (a) Change of Alpha with increasing process duration of measurement series A and B; (b) NIR-spectra (raw data) of series A.

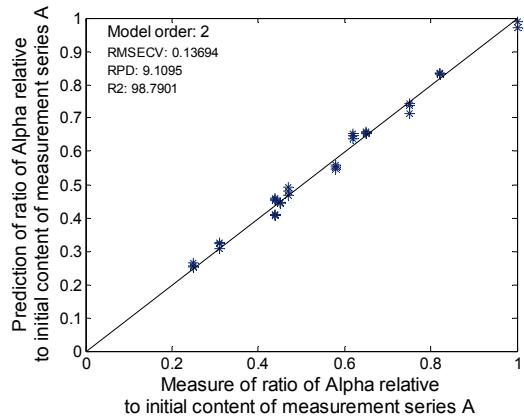

(a)

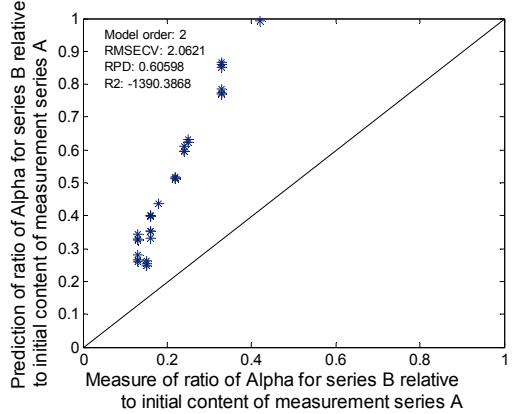

(b)

Figure 4: (a) Calculation of the calibration model of measurement series A;

(b) Application to series B for absolute values of Alpha.

measurement series. This results partly in a determination of the auxiliary quantity by extrapolation which leads to a poor fit. In addition, the rate of change of Alpha varies significantly due to the different initial compositions.

Figure 5(a) represents the calculation of the calibration model with the relative change of Alpha with respect to the working point for measurement series A. The best results in terms of criteria of fit were achieved using the same combination of data pre-processing for both calculation of the calibration model with the absolute values and calculation with the relative values. The best spectral range and the selected model order are identical as well. Calculations of the calibration model and of the cross-validation result in relatively good values for $\mathrm{R}^{2}(98.8 \%)$ and the relative error in average (3.91\%).

Through the implementation of this method a significant improvement of the results can be achieved when applying the calculated calibration model of measurement series A to measurement series B, which is shown in Figure 5(b). When the calibration model is then applied to the new measurement series the 
rate of change is predicted, which can be recalculated to an absolute value for Alpha with the known initial value (working point) of the measurement series. The application of the calibration model results in a $\mathrm{R}^{2}$ of all the measured spectra of $96.0 \%$ and a relative error in average of $7.69 \%$. Even for the values laying outside the calibration range a good prediction could be achieved by this procedure.

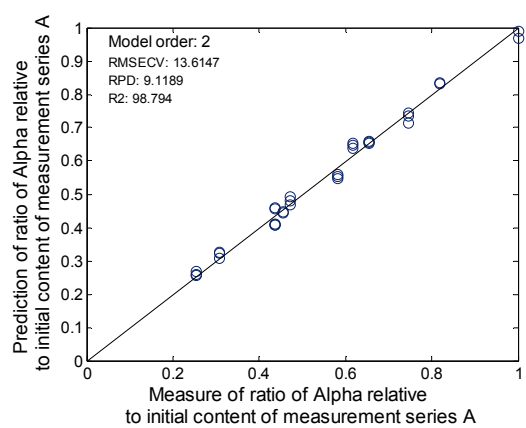

(a)

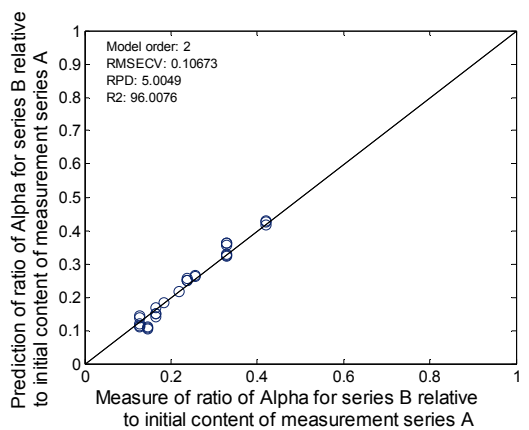

(b)

Figure 5: (a) Calculation of the calibration model of series A; (b) Application to series $\mathrm{B}$ for adaptation of the target vector to the working point with relative values recalculated to absolute values of Alpha.

This new method has been applied for all defined auxiliary quantities (fraction of Alpha in the sample, crystallite size of the quartz crystals and fraction amorphous material in the sample). Alpha showed significantly better results for the application of the adaptation to the working point by the introduction of the relative values. An improved or at least respectively equally good calibration model was calculated with this established relative values for auxiliary quantities sizeQuartz and Amorphous. The application to the new measurement series and assessment with cross-validation shows also equally good or even better results for these two auxiliary quantities.

\section{Conclusions}

The process control of the manufacture of the novel cementitious material Celitement is a challenging task mainly due to its X-ray amorphous nature. In order to develop suitable prediction of the product quality based on fast NIR-spectroscopy, different auxiliary quantities had to be identified. In an initial attempt three auxiliary parameters were considered: the fraction of amorphous material in the sample, the proportion of $\alpha-\mathrm{C}_{2} \mathrm{SH}$ in the sample and the crystallite size of the quartz crystals included in the product. It was shown that these auxiliary quantities can be measured by FT-NIR-spectroscopy applying suitable calibration models. During the preparation of the calibration models different alternatives of data pre-processing, subdivision of the spectra into several spectral ranges and selection of various model orders were considered. The calibration models were also calculated on the one hand for the absolute values 
of the auxiliary quantities and on the other hand for a relative value related to the initial value (working point). Whereas good results could be achieved for the auxiliary quantities Amorphous (fraction of X-ray-amorphous material in the sample) and sizeQuarz (the crystallite size of the quartz crystals) using the absolute values for the calculation of the calibration model and subsequent application to new measurement series, this method could not be applied for the determination of $\alpha-\mathrm{C}_{2} \mathrm{SH}$ in the sample. The calculation of the calibration model with adaptation of the target vector to the working point provided significantly better results. Despite different initial compositions of the sample series, the application of the calibration model to a new measurement series was successfully implemented using this method. In a further step, it will be necessary to represent the product quality quantitatively through various auxiliary quantities and to calculate the corresponding calibration models for multiple records of measurement series. Subsequently, the on-line determination of the product quality by defined auxiliary quantities can be integrated in the process control.

\section{References}

[1] Olivier, J.G.J., Janssens-Maenhout, G. \& Peters, J.A.H.W.: Trends in global $\mathrm{CO}_{2}$ emissions. PBL Netherlands Environmental Assessment Agency, 2012.

[2] Celitement GmbH, http://www.Celitement.de/en/

[3] Stemmermann, P., Schweike, U., Garbev, K. \& Beuchle, G.: Celitement a sustainable prospect for the cement. CEMENT INTERNATIONAL, pp. 52-66, 2010.

[4] G. Beuchle, P. Stemmermann, U. Schweike, K. Garbev: Single-phase hydraulic binder, methods for the production thereof and building material produced therewith, Grant, 2008.

[5] Burns, D.A. \& Ciurczak, E.W.: Handbook of near-infrared analysis. New York: Marcel Dekker, 1992.

[6] Hollas, J. M.: Modern Spectroscopy. West Sussex, England: John Wiley \& Sons, 2004.

[7] Herres, W. \& Gronholz, J.: Understanding FT-IR Data Processing. Bruker Analytische Messtechnik GmbH, Karlsruhe, 1984.

[8] Bruker Corporation, http://www.bruker.com/

[9] BRUKER OPTIK GmbH: OPUS Spectroscopy Software - User Manual QUANT. Ettlingen: BRUKER OPTIK GmbH, 2006.

[10] Barnes, R.J, Dhanoa, M.S. \& Lister, S.J.: Standard Normal Variate Transformation and De-trending of Near-Infrared Diffuse Reflectance Spectra. Applied Spectroscopy. 5, Vol. 43, 1989.

[11] Martens, H. \& Naes, T.: Multivariate calibration. Chichester: Wiley, 1989.

[12] Ruscio, D.D.: A weighted view on the partial least-squares algorithm. Automatica, Vol. 36, 6, 2000. 